\title{
Comparison Between Reverberation-Ray Matrix, Reverberation-Transfer Matrix, and Generalized Reverberation Matrix
}

\author{
Jiayong Tian \\ Institute of Crustal Dynamics, China Earthquake Administration \\ P.R.China
}

\section{Introduction}

Different matrix formulations have been developed to investigate the elastic-wave propagation in a multilayered solid, which have been widely used in the fields of seismology, ocean acoustics(Pao et al., 2000), and non-destructive evaluation (Lowe, 1995), etc. Transfer matrix (TM) method (Haskell, 1953; Thomson, 1950), as one of the most important matrix formulations, yields a simple configuration and efficient computational ability to facilitate its wide application in many research fields. Stiffness matrix (SM) method (Rokhlin \& Wang, 2002; Wang \& Rokhlin, 2001) has been proposed to resolve the inherent computational instability for the large product of frequency and thickness in TM method. The SM formulation utilizes the stiffness matrix of each sublayer in a recursive algorithm to obtain the stacked stiffness matrix for the multilayered solid. However, the SM formulation is difficult to identify the generalized-ray propagation in the multilayered solid.

In order to evaluate the transient wave propagation in the multilayered solid, $\mathrm{Su}$, Tian, and Pao (Su et al., 2002; Tian \& Xie, 2009; Tian et al., 2006) presented reverberation-ray matrix (RRM) formulation. Introducing the local scattering relations at interfaces and the phase relations in sublayers, a system of equations is formulated by a reverberation matrix $\mathbf{R}$, which can be automatically represented as a series of generalized ray group integrals according to the times of reflections and refractions of generalized rays at interfaces. Each generalized ray group integral containing $\mathbf{R}^{k}$ represents the set of $K$ times reflections and transmissions of source waves arriving at receivers in the multilayered solid, which is very suitable to automatic computer programming for the simple multilayered-solid configuration. However, the dimension of the reverberation matrix will increase as the number of the sublayers increases, which may yield the lower calculation efficiency of the generalized-ray groups in the complex multilayered solid(Tian \& Xie, 2009).

In order to increase the calculation efficiency of the generalized-ray groups, Tian presented the reverberation-transfer matrix (RTM) and generalized reverberation matrix (GRM) formulations, respectively. In RTM formulation, RRM formulation is applied to the interested sublayer for the evaluation of the generalized rays and TM formulation to the other sublayers, to construct a RTM of the constant dimension, which is independent of the sublayer number. However, the RTM suffers from the inherent numerical instabilities particularly when the layer thickness becomes large and/ or the frequency is high. GRM 
formulation is to integrate RRM and SM formulations. The RRM formulation is applied to the interested sublayer for the evaluation of the generalized rays and SM formulation to the other sublayers, to construct a generalized reverberation matrix of the constant dimension, which is independent of the sublayer number. GRM formulation has the higher calculation efficiency and numerical stabilities of the generalized rays in the complex multilayered-solid configuration.

In this chapter, in order to facilitate the wide application of RRM, RTM, and GRM formulations, we compare them clearly to show their difference and applicability.

\section{RRM formulation}

Here, we only consider in-plane wave propagation in a laminate containing $N$ isotropic sublayers impacted by the vertical force $f(t, x)$ on the top surface of the laminate. In RRM formulation, the interfaces between sublayers are expressed by capital letters $I, J, \cdots$. Two local Cartesian coordinate systems $(x, y)^{I I}$ and $(x, y)^{I I}$ are constructed at two interfaces of sublayer IJ, respectively. The thickness of sublayer IJ is represented by $h^{I I}$, which is shown in Fig.1.
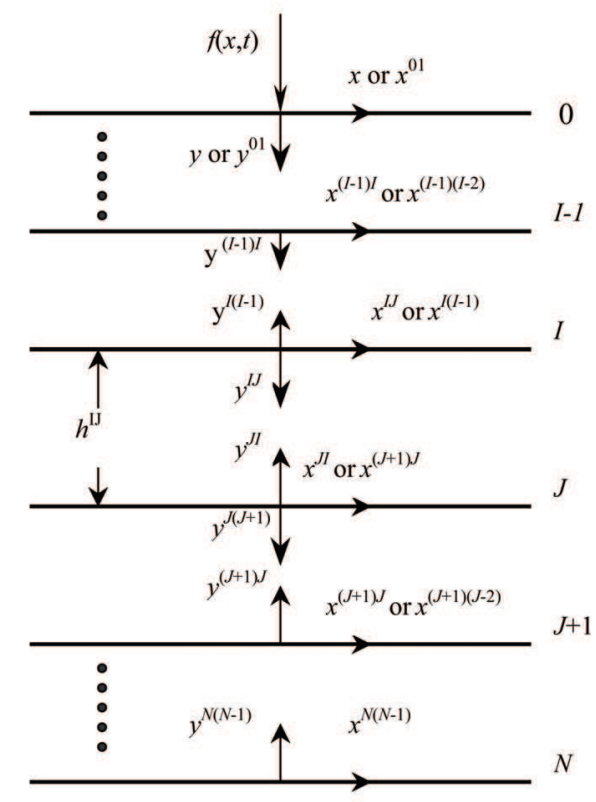

Fig. 1. The schematic diagram of the global and local coordinates for RRM formulation

Introducing the one-side Laplace transform with respect to the time and the double-side Laplace transform with respect to the spatial variable $x$ as(Achenbach, 1973)

$$
\hat{f}(\eta, y, p)=\int_{-\infty}^{\infty} e^{-p \eta x} \int_{0}^{\infty} f(x, y, t) e^{-p t} d t d x
$$

the transformed wave equations with related to the displacement potentials $\varphi^{\text {II }}$ and $\psi^{\text {II }}$ in the local Cartesian coordinate system $(x, y)^{I J}$ are denoted as 


$$
\left.\begin{array}{l}
\frac{\partial^{2} \varphi^{I I}}{\partial y^{2}}-\left(\gamma_{1}^{I I}\right)^{2} \varphi^{I I}=0 \\
\frac{\partial^{2} \psi^{I I}}{\partial y^{2}}-\left(\gamma_{2}^{I I}\right)^{2} \psi^{I I}=0
\end{array}\right\},
$$

Introduction of the unknown arriving and departing wave-amplitude vectors $\hat{\mathbf{a}}^{I I}=\left\{a_{1}^{I I}, a_{2}^{I I}\right\}^{T}$, $\hat{\mathbf{d}}^{I J}=\left\{d_{1}^{I I}, d_{2}^{I I}\right\}^{T}$ in the local coordinate system $(x, y)^{I J}$ yields the displacement and stress vectors $\hat{\mathbf{U}}^{I J}\left(\eta, y^{I I}, p\right)=\left\{\hat{u}_{x}^{I J}, \hat{u}_{y}^{I I}\right\}^{T}, \hat{\mathbf{F}}^{I J}\left(\eta, y^{I J}, p\right)=\left\{\hat{\tau}_{y x}^{I I}, \hat{\tau}_{y y}^{I I}\right\}^{T}$ from Eq. (2) as

$$
\left.\begin{array}{l}
\hat{\mathbf{U}}^{I I}\left(\eta, y^{I J}, p\right)=p \mathbf{A}_{u}^{I I} \hat{\mathbf{a}}^{I I}+p \mathbf{D}_{u}^{I J} \hat{\mathbf{d}}^{I I} \\
\hat{\mathbf{F}}^{I I}\left(\eta, y^{I I}, p\right)=\mu^{I I} p^{2} \mathbf{A}_{f}^{I I} \hat{\mathbf{a}}^{I I}+\mu^{I I} p^{2} \mathbf{D}_{f}^{I I} \hat{\mathbf{d}}^{I J}
\end{array}\right\},
$$

where $\mathbf{A}_{u}^{I I}$ and $\mathbf{D}_{u}^{I I}$ are phase-related receiver matrixes for the displacements, $\mathbf{A}_{f}^{I J}$ and $\mathbf{D}_{f}^{I I}$ for the stresses, respectively. With the definition of the arriving wave amplitude vector $\mathbf{a}^{J}$ and the departing wave amplitude vector $\mathbf{d}^{J}$ of interface $J$ as

$$
\begin{aligned}
& \mathbf{a}^{J}=\left\{a_{1}^{J(J-1)}, a_{2}^{J(J-1)}, a_{1}^{J(J+1)}, a_{2}^{J(J+1)}\right\}^{T} \\
& \left.\mathbf{d}^{J}=\left\{d_{1}^{J(J-1)}, d_{2}^{J(J-1)}, d_{1}^{J(J+1)}, d_{2}^{J(J+1)}\right\}^{T}\right\},
\end{aligned}
$$

the application of the boundary conditions yields scattering relation at interface $J$

$$
\mathbf{d}^{J}=\mathbf{S}^{J} \mathbf{a}^{J}+\mathbf{s}^{J},
$$

where $\mathbf{S}^{J}$ and $\mathbf{s}^{J}$ are the scattering matrix and source matrix of interface $J$, respectively. With the definition of global arriving and departing wave amplitude vectors $\mathbf{a}=\left\{\left\{\mathbf{a}^{1}\right\}^{T},\left\{\mathbf{a}^{2}\right\}^{T}, \ldots,\left\{\mathbf{a}^{N}\right\}^{T}\right\}^{T}$ and $\mathbf{d}=\left\{\left\{\mathbf{d}^{1}\right\}^{T},\left\{\mathbf{d}^{2}\right\}^{T}, \ldots,\left\{\mathbf{d}^{N}\right\}^{T}\right\}^{T}$, the global scattering matrix can be written in the following form

$$
\mathbf{d}=\mathbf{S a}+\mathbf{s} .
$$

Since both vectors $\mathbf{a}$ and $\mathbf{d}$ are unknown quantities, an additional equation related to $\mathbf{a}$ and $\mathbf{d}$ must be provided. A wave arriving at interface $I$ in the local coordinate $(x, y)^{I J}$, is also considered as the wave departing from interface $J$ of the same layer in the local coordinate $(x, y)^{I I}$, which yields the other relation between the global arriving and departing wave amplitude vectors

$$
\mathbf{a}=\text { PHd , }
$$

where the phase matrix $\mathbf{P}$ is a $4 N \times 4 N$ diagonal matrix. $\mathbf{H}$ is a $4 N \times 4 N$ matrix composed of only one element whose value is one in each line and each row and others are all zero. For example, in vector $\mathbf{d}$, if $d_{i}^{\zeta K}$ and $d_{i}^{K J}$ are in the positions $p$ and $q$ respectively, then the elements $H_{p q}$ and $H_{q p}$ in the matrix $\mathbf{H}$ have the same value one. 


\section{RTM and GRM formulations}

In RTM and GRM formulations, assuming that the receiver is in the sublayer IJ, the laminate can be partitioned into three layers, which includes layers OI, IJ, and JN as shown in Fig.2. If the receiver is in the top or bottom sublayer, the laminate will be partitioned into two layers, which includes layers 01 , and $1 N$, or $1(N-1)$ and $(N-1) N$, respectively.
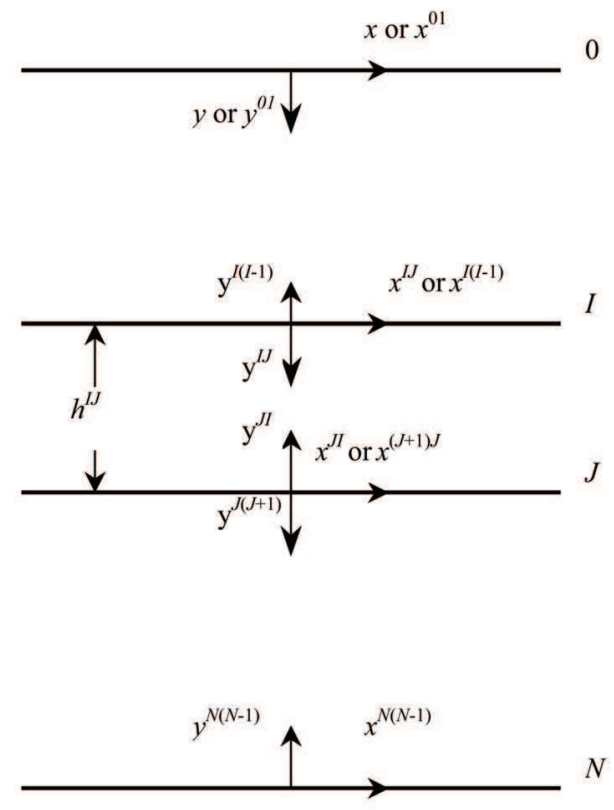

Fig. 2. The schematic diagram of the global and local coordinates for RRM formulation

Considering the continuity conditions at the interfaces $I$ and $J$ and boundary conditions at the surfaces, the scattering relations at interfaces $0, I, J$, and $N$ yield,

$$
\left.\begin{array}{l}
\mathbf{d}^{0}=\mathbf{S}^{0} \mathbf{a}^{0}+\mathbf{s}^{0} \\
\mathbf{d}^{I}=\mathbf{S}^{I} \mathbf{a}^{I}+\mathbf{s}^{I} \\
\mathbf{d}^{I}=\mathbf{S}^{J} \mathbf{a}^{I}+\mathbf{s}^{I} \\
\mathbf{d}^{N}=\mathbf{S}^{N} \mathbf{a}^{N}+\mathbf{s}^{N}
\end{array}\right\} .
$$

If we stack all the local amplitude vectors of the arriving and departing waves as $\mathbf{a}=\left\{\left\{\mathbf{a}^{0}\right\}^{T},\left\{\mathbf{a}^{I}\right\}^{T},\left\{\mathbf{a}^{I}\right\}^{T},\left\{\mathbf{a}^{N}\right\}^{T}\right\}^{T}$ and $\mathbf{d}=\left\{\left\{\mathbf{d}^{0}\right\}^{T},\left\{\mathbf{d}^{I}\right\}^{T},\left\{\mathbf{d}^{J}\right\}^{T},\left\{\mathbf{d}^{N}\right\}^{T}\right\}^{T}$, the global scattering relation can be denoted as

$$
\mathbf{d}=\mathbf{S a}+\mathbf{s}
$$

where $\mathbf{S}$ and $\mathbf{s}$ are the global scattering matrix and source matrix, respectively. Since both a and $\mathbf{d}$ are unknown quantities, an additional equation related to $\mathbf{a}$ and $\mathbf{d}$ must be provided. 


\subsection{Sublayer IJ}

Similar with the RRM formulation, the local phase matrix for the sublayers $I J$ can be given as

$$
\left.\begin{array}{l}
\mathbf{a}^{I I}=\mathbf{P}^{I I} \mathbf{d}^{I I} \\
\mathbf{a}^{I I}=\mathbf{P}^{I I} \mathbf{d}^{I J}
\end{array}\right\}
$$

\subsection{Layers $0 I$ and $J N$}

\subsubsection{Reverberation-transfer matrix formulation}

In layers $0 I$ or $J N, T M$ formulation is adopted to describe the relations of wave amplitudes between interfaces 0 and $I$ or interfaces $J$ and $N$. The displacements and stresses at interfaces 0 and 1 in the local coordinate $(x, y)^{01}$ are written as

$$
\left.\begin{array}{l}
\hat{\mathbf{G}}^{01}(0)=\mathbf{C}^{01} \mathbf{b}^{01} \\
\hat{\mathbf{G}}^{01}\left(h^{01}\right)=\mathbf{E}^{01} \mathbf{b}^{01}
\end{array}\right\},
$$

where $\mathbf{G}^{01}\left(y^{01}\right)=\left\{\left\{\hat{\mathbf{U}}\left(\eta, y^{01}, p\right)\right\}^{T},\left\{\hat{\mathbf{F}}\left(\eta, y^{01}, p\right)\right\}^{T}\right\}, \quad \mathbf{b}^{01}=\left\{\left\{\mathbf{a}^{01}\right\}^{T},\left\{\mathbf{d}^{01}\right\}^{T}\right\}^{T}$. Hence, their relations are deduced from the above equations

$$
\hat{\mathbf{G}}^{01}\left(h^{01}\right)=\mathbf{E}^{01}\left(\mathbf{C}^{01}\right)^{-1} \hat{\mathbf{G}}^{01}(0) .
$$

Using the above recursion, we obtain the displacement-stress relation between interfaces 0 and $I$,

$$
\hat{\mathbf{G}}^{(I-1) I}\left(h^{(I-1) I}\right)=\left(\prod_{k=1}^{I} \mathbf{E}^{(k-1) k}\left(\mathbf{C}^{(k-1) k}\right)^{-1}\right) \hat{\mathbf{G}}^{01}(0) .
$$

The displacements and stresses at interface $I$ in the local coordinate $(x, y)^{I(I-1)}$ can be denoted as

$$
\hat{\mathbf{G}}^{I(I-1)}(0)=\mathbf{C}^{I(I-1)} \mathbf{b}^{I(I-1)} .
$$

Here, $\hat{\mathbf{G}}^{I(I-1)}(0)$ and $\hat{\mathbf{G}}^{(I-1) I}\left(h^{(I-1) I}\right)$ represent the displacements and stresses at the same position but in the different local coordinate systems, which have the following relation as,

$$
\hat{\mathbf{G}}^{I(I-1)}(0)=\mathbf{T} \hat{\mathbf{G}}^{(I-1) I}\left(h^{(I-1) I}\right),
$$

where $\mathbf{T}=\operatorname{diag}\{1,-1,-1,1\}$. Substitution of Eqs. (11-14) into Eq.(15) yields

$$
\mathbf{b}^{I(I-1)}=\mathbf{L b}^{01},
$$

where $\mathbf{L}=\left(\mathbf{C}^{I(I-1)}\right)^{-1} \mathbf{T}\left(\prod_{k=1}^{I} \mathbf{E}^{(k-1) k}\left(\mathbf{C}^{(k-1) k}\right)^{-1}\right) \mathbf{C}^{01} \cdot \mathbf{b}^{01}$ and $\mathbf{b}^{I(I-1)}$ represent wave amplitudes at interfaces 0 and $I$ in the local coordinate $(x, y)^{01}$ and $(x, y)^{I(I-1)}$, respectively. Separation of Eq.(26) into the arriving and departing waves yields 


$$
\left[\begin{array}{c}
\mathbf{a}^{01} \\
\mathbf{a}^{I(I-1)}
\end{array}\right]=\mathbf{P}^{0 I}\left[\begin{array}{c}
\mathbf{d}^{I(I-1)} \\
\mathbf{d}^{01}
\end{array}\right]
$$

where $\mathbf{P}^{0 I}=\left[\begin{array}{ll}-\mathbf{L}_{11} & \mathbf{I} \\ -\mathbf{L}_{21} & \mathbf{0}\end{array}\right]^{-1}\left[\begin{array}{cc}\mathbf{0} & \mathbf{L}_{12} \\ -\mathbf{I} & \mathbf{L}_{22}\end{array}\right]$ is the phase matrix of layer $0 I$, and $\mathrm{I}$ is a $2 \times 2$ identity matrix. Similarly, the phase relation of the arriving and departing waves in layer $J N$ can be denoted as

$$
\left[\begin{array}{c}
\mathbf{a}^{J(J+1)} \\
\mathbf{a}^{N(N-1)}
\end{array}\right]=\mathbf{P}^{J N}\left[\begin{array}{l}
\mathbf{d}^{N(N-1)} \\
\mathbf{d}^{J(+1)}
\end{array}\right]
$$

\subsubsection{GRM formulation}

In layer $0 I$ or $J N$, stiffness matrix formulation is adopted to describe the relations of wave amplitudes between interfaces 0 and $I$ or interfaces $J$ and $N$. The displacements and stresses at interfaces 0 and 1 in the local coordinate system $(x, y)^{01}$ are written as

$$
\begin{aligned}
& {\left[\begin{array}{c}
\hat{\mathbf{U}}\left(\eta, 0^{01}, p\right) \\
\hat{\mathbf{U}}\left(\eta, h^{01}, p\right)
\end{array}\right]=\mathbf{E}_{u}^{01} \mathbf{b}^{01},} \\
& {\left[\begin{array}{c}
\hat{\mathbf{F}}\left(\eta, 0^{01}, p\right) \\
\hat{\mathbf{F}}\left(\eta, h^{01}, p\right)
\end{array}\right]=\mathbf{E}_{f}^{01} \mathbf{b}^{01},}
\end{aligned}
$$

where

$$
\begin{gathered}
\mathbf{E}_{f}^{01}=\mu^{01} p^{2}\left[\begin{array}{ll}
\mathbf{A}_{f}^{01}\left(0^{01}\right) & \mathbf{D}_{f}^{01}\left(0^{01}\right) \\
\mathbf{A}_{f}^{01}\left(h^{01}\right) & \mathbf{D}_{f}^{01}\left(h^{01}\right)
\end{array}\right], \\
\mathbf{E}_{u}^{01}=p\left[\begin{array}{ll}
\mathbf{A}_{u}^{01}\left(0^{01}\right) & \mathbf{D}_{u}^{01}\left(0^{01}\right) \\
\mathbf{A}_{u}^{01}\left(h^{01}\right) & \mathbf{D}_{u}^{01}\left(h^{01}\right)
\end{array}\right], \\
\mathbf{b}^{01}=\left\{\left[\mathbf{a}^{01}\right]^{T},\left[\mathbf{d}^{01}\right]^{T}\right\}^{T} .
\end{gathered}
$$

The substitution of $\mathbf{b}^{01}$ from Eq. (19) into Eq. (20) yields the stiffness $\mathbf{K}^{01}$ of sublayer 01

$$
\left[\begin{array}{c}
\hat{\mathbf{F}}\left(\eta, 0^{01}, p\right) \\
\hat{\mathbf{F}}\left(\eta, h^{01}, p\right)
\end{array}\right]=\left[\begin{array}{ll}
\mathbf{K}_{11}^{01} & \mathbf{K}_{12}^{01} \\
\mathbf{K}_{21}^{01} & \mathbf{K}_{22}^{01}
\end{array}\right]\left[\begin{array}{c}
\hat{\mathbf{U}}\left(\eta, 0^{01}, p\right) \\
\hat{\mathbf{U}}\left(\eta, h^{01}, p\right)
\end{array}\right]
$$

where $\mathbf{K}^{01}=\mathbf{E}_{f}^{01}\left(\mathbf{E}_{u}^{01}\right)^{-1}$. Similarly, the stiffness $\mathbf{K}^{12}$ of sublayer 12 can be denoted as

$$
\left[\begin{array}{l}
\hat{\mathbf{F}}\left(\eta, 0^{12}, p\right) \\
\hat{\mathbf{F}}\left(\eta, h^{12}, p\right)
\end{array}\right]=\left[\begin{array}{ll}
\mathbf{K}_{11}^{12} & \mathbf{K}_{12}^{12} \\
\mathbf{K}_{21}^{12} & \mathbf{K}_{22}^{12}
\end{array}\right]\left[\begin{array}{c}
\hat{\mathbf{U}}\left(\eta, 0^{12}, p\right) \\
\hat{\mathbf{U}}\left(\eta, h^{12}, p\right)
\end{array}\right]
$$


The stiffness $\mathbf{K}^{02}$ of layer 02 can be deduced from Eqs. (21) and (22) as

$$
\left[\begin{array}{c}
\hat{\mathbf{F}}\left(\eta, 0^{01}, p\right) \\
\hat{\mathbf{F}}\left(\eta, h^{12}, p\right)
\end{array}\right]=\mathbf{K}^{02}\left[\begin{array}{c}
\hat{\mathbf{U}}\left(\eta, 0^{01}, p\right) \\
\hat{\mathbf{U}}\left(\eta, h^{12}, p\right)
\end{array}\right]
$$

where

$$
\mathbf{K}^{02}=\left[\begin{array}{cc}
\mathbf{K}_{11}^{01}+\mathbf{K}_{12}^{01}\left(\mathbf{K}_{11}^{12}-\mathbf{K}_{22}^{01}\right)^{-1} \mathbf{K}_{21}^{01} & -\mathbf{K}_{12}^{01}\left(\mathbf{K}_{11}^{12}-\mathbf{K}_{22}^{01}\right)^{-1} \mathbf{K}_{12}^{12} \\
\mathbf{K}_{21}^{12}\left(\mathbf{K}_{11}^{12}-\mathbf{K}_{22}^{01}\right)^{-1} \mathbf{K}_{21}^{01} & \mathbf{K}_{22}^{12}-\mathbf{K}_{21}^{12}\left(\mathbf{K}_{11}^{12}-\mathbf{K}_{22}^{01}\right)^{-1} \mathbf{K}_{12}^{12}
\end{array}\right]
$$

The stiffness $\mathbf{K}^{0 I}$ of layer $0 I$ can be deduced from the above recursion as

$$
\left[\begin{array}{c}
\hat{\mathbf{F}}\left(\eta, 0^{01}, p\right) \\
\hat{\mathbf{F}}\left(\eta, h^{(I-1) I}, p\right)
\end{array}\right]=\mathbf{K}^{0 I}\left[\begin{array}{c}
\hat{\mathbf{U}}\left(\eta, 0^{01}, p\right) \\
\hat{\mathbf{U}}\left(\eta, h^{(I-1) I}, p\right)
\end{array}\right]
$$

where

$$
\mathbf{K}^{0 I}=\left[\begin{array}{cc}
\mathbf{K}_{11}^{0(I-1)}+\mathbf{K}_{12}^{0(I-1)}\left(\mathbf{K}_{11}^{(I-1) I}-\mathbf{K}_{22}^{0(I-1)}\right)^{-1} \mathbf{K}_{21}^{0(I-1)} & -\mathbf{K}_{12}^{0(I-1)}\left(\mathbf{K}_{11}^{(I-1) I}-\mathbf{K}_{22}^{0(I-1)}\right)^{-1} \mathbf{K}_{12}^{(I-1) I} \\
\mathbf{K}_{21}^{(I-1) I}\left(\mathbf{K}_{11}^{(I-1) I}-\mathbf{K}_{22}^{0(I-1)}\right)^{-1} \mathbf{K}_{21}^{0(I-1)} & \mathbf{K}_{22}^{(I-1) I}-\mathbf{K}_{21}^{(I-1) I}\left(\mathbf{K}_{11}^{(I-1) I}-\mathbf{K}_{22}^{0(I-1)}\right)^{-1} \mathbf{K}_{12}^{(I-1) I}
\end{array}\right] .
$$

The displacements and stresses at interface $I$ in the local coordinate system $(x, y)^{(I-1) I}$ can be denoted in the local coordinate system $(x, y)^{I(I-1)}$ as

$$
\begin{aligned}
& {\left[\begin{array}{c}
\hat{\mathbf{F}}\left(\eta, 0^{01}, p\right) \\
\hat{\mathbf{F}}\left(\eta, h^{(I-1) I}, p\right)
\end{array}\right]=\mathbf{T}_{f}\left[\begin{array}{c}
\hat{\mathbf{F}}\left(\eta, 0^{01}, p\right) \\
\hat{\mathbf{F}}\left(\eta, 0^{I(I-1)}, p\right)
\end{array}\right],} \\
& {\left[\begin{array}{c}
\hat{\mathbf{U}}\left(\eta, 0^{01}, p\right) \\
\hat{\mathbf{U}}\left(\eta, h^{(I-1) I}, p\right)
\end{array}\right]=\mathbf{T}_{u}\left[\begin{array}{c}
\hat{\mathbf{U}}\left(\eta, 0^{01}, p\right) \\
\hat{\mathbf{U}}\left(\eta, 0^{I(I-1)}, p\right)
\end{array}\right] .}
\end{aligned}
$$

where $\mathbf{T}_{f}=\operatorname{diag}\{1,1,-1,1\}, \mathbf{T}_{u}=\operatorname{diag}\{1,1,1,-1\}$. Substitution of Eqs. (25) and (26) into Eq. (24) yields

$$
\left[\begin{array}{c}
\hat{\mathbf{F}}\left(\eta, 0^{01}, p\right) \\
\hat{\mathbf{F}}\left(\eta, 0^{I(I-1)}, p\right)
\end{array}\right]=\overline{\mathbf{K}}^{0 I}\left[\begin{array}{c}
\hat{\mathbf{U}}\left(\eta, 0^{01}, p\right) \\
\hat{\mathbf{U}}\left(\eta, 0^{I(I-1)}, p\right)
\end{array}\right]
$$

where $\overline{\mathbf{K}}^{01}=\left(\mathbf{T}_{f}\right)^{-1} \mathbf{K}^{01} \mathbf{T}_{u}$. Equation (27) can be expressed by the arriving-wave and departing-wave amplitude vectors,

$$
\left[\begin{array}{ll}
\mathbf{A}_{f} & \mathbf{D}_{f}
\end{array}\right]\left[\begin{array}{c}
\mathbf{a}^{01} \\
\mathbf{a}^{I(I-1)} \\
\mathbf{d}^{I(I-1)} \\
\mathbf{d}^{01}
\end{array}\right]=\overline{\mathbf{K}}^{0 I}\left[\begin{array}{ll}
\mathbf{A}_{u} & \mathbf{D}_{u}
\end{array}\right]\left[\begin{array}{c}
\mathbf{a}^{01} \\
\mathbf{a}^{I(I-1)} \\
\mathbf{d}^{I(I-1)} \\
\mathbf{d}^{01}
\end{array}\right],
$$


where

$$
\begin{aligned}
& \mathbf{A}_{f}=\left[\begin{array}{cc}
\mathbf{A}_{f}^{01}\left(0^{01}\right) & \mathbf{0} \\
\mathbf{0} & \mathbf{A}_{f}^{I(I-1)}\left(0^{I(I-1)}\right)
\end{array}\right], \\
& \mathbf{D}_{f}=\left[\begin{array}{cc}
\mathbf{0} & \mathbf{D}_{f}^{01}\left(0^{01}\right) \\
\mathbf{D}_{f}^{I(I-1)}\left(0^{I(I-1)}\right) & \mathbf{0}
\end{array}\right], \\
& \mathbf{A}_{u}=\left[\begin{array}{cc}
\mathbf{A}_{u}^{01}\left(0^{01}\right) & \mathbf{0} \\
\mathbf{0} & \mathbf{A}_{u}^{I(I-1)}\left(0^{I(I-1)}\right)
\end{array}\right], \\
& \mathbf{D}_{u}=\left[\begin{array}{cc}
\mathbf{0} & \mathbf{D}_{u}^{01}\left(0^{01}\right) \\
\mathbf{D}_{u}^{I(I-1)}\left(0^{I I I-1)}\right) & \mathbf{0}
\end{array}\right] .
\end{aligned}
$$

Separation of Eq. (28) into the wave-amplitude vectors of the arriving and departing waves results in

$$
\left[\begin{array}{c}
\mathbf{a}^{01} \\
\mathbf{a}^{I(I-1)}
\end{array}\right]=\mathbf{P}^{0 I}\left[\begin{array}{c}
\mathbf{d}^{I(I-1)} \\
\mathbf{d}^{01}
\end{array}\right],
$$

where $\mathbf{P}^{0 I}=\left[\mathbf{A}_{f}-\overline{\mathbf{K}}^{0 I} \mathbf{A}_{u}\right]^{-1}\left[\overline{\mathbf{K}}^{0 I} \mathbf{D}_{u}-\mathbf{D}_{f}\right]$ is the phase matrix of layer 0I. Similarly, the phase relation of the arriving and departing waves in layer JN can be denoted as

$$
\left[\begin{array}{c}
\mathbf{a}^{J(J+1)} \\
\mathbf{a}^{N(N-1)}
\end{array}\right]=\mathbf{P}^{J N}\left[\begin{array}{c}
\mathbf{d}^{N(N-1)} \\
\mathbf{d}^{J(J+1)}
\end{array}\right] .
$$

\section{Expansion of generalized-ray groups}

The unknow amplitude vectors a and $\mathbf{d}$ in RRM, RTM, and GRM formulations yields

$$
\left.\begin{array}{l}
\mathbf{d}=[\mathbf{I}-\mathbf{R}]^{-1} \mathbf{s} \\
\mathbf{a}=\mathbf{P H}[\mathbf{I}-\mathbf{R}]^{-1} \mathbf{s}
\end{array}\right\},
$$

where $\mathbf{R}=\mathbf{S P H}$ is reverberation matrix. Once $\mathbf{d}$ and $\mathbf{a}$ are known, the transformed displacements can be denoted as

$$
\hat{\mathbf{U}}\left(\eta, y^{I I}, p\right)=p\left(\mathbf{A}_{u} \mathbf{P H}+\mathbf{D}_{u}\right)(\mathbf{I}-\mathbf{R})^{-1} \mathbf{s} .
$$

The transient displacements can be expressed by applying the inverse transforms

$$
\mathbf{U}\left(x, y^{I J}, t\right)=\frac{1}{2 \pi i} \int_{0}^{\infty} \int_{B r} p^{2}\left(\mathbf{A}_{u} \mathbf{P H}+\mathbf{D}_{u}\right)(\mathbf{I}-\mathbf{R})^{-1} \mathbf{s} e^{p t} e^{p \eta x} d p d \eta
$$


The replacement of the matrix $[\mathbf{I}-\mathbf{R}]^{-1}$ by the power series $\left[\mathbf{I}+\mathbf{R}+\mathbf{R}^{2}+\ldots+\mathbf{R}^{k}+\ldots\right]$ rewrites Eq. (33) as

$$
\mathbf{U}\left(x, y^{I I}, t\right)=\sum_{k=0}^{\infty} \frac{1}{2 \pi i} \int_{0}^{\infty} \int_{B r} p^{2}\left(\mathbf{A}_{u} \mathbf{P H}+\mathbf{D}_{u}\right) \mathbf{R}^{k} \mathbf{s} e^{p t} e^{p \eta x} d p d \eta,
$$

where the double integrals can be evaluated by fast Fourier transform (FFT) formulation (Tian \& Xie, 2009).

\section{Comparisons and discussions}

Equation (31) shows that RRM, RTM, and GRM formulations have the same expression of reverberation matrix $\mathbf{R}$. In RTM and GRM formulations, the dimension of $\mathbf{R}$ is in general $12 \times 12$, which is independent of the sublayer number. If the receiver is in the top or bottom sublayer, reverberation matrix has the order of $8 \times 8$. However, $\mathbf{R}$ in RRM formulation is a $4 N \times 4 N$ matrix, which means that the calculation efficiency of RRM formulation will decrease as the sublayer number increases.

Each term of the double integral in Eq.(34) containing $\mathbf{R}^{k}$ are defined as a generalized ray group. In RRM formulation, a generalized ray group represents the set of $k$ times reflections and transmissions of the source waves by all interfaces arriving at receivers at $(x, y)$. When $k=0$, the genralized ray group shows the waves from sources to the receivers directly without any reflection or refraction, which are called as source waves. Here, every generalized ray group contains a series of generalized rays, and the number of generalized rays increases exponentially with the increase of the number of layer and the reflection or refraction times. However, In RTM and GRM formulations, a generalized ray group represents the set of the generalized rays arriving at receiver $(x, y)$ with $k$ times of reflections and transmissions by interfaces $0, I, J$, and $N$.

In RTM formulation, the reverberation matrix $\mathbf{R}$ has the inherent computational instability for the large product of frequency and thickness, which means that RTM formulation only can be applied to the investigation of low-frequency wave propagation in the multilayered solid. However, RRM and GRM can promise the numerical stability for the large product of frequency and thickness.

In the following, we validate the calculation efficiency of RRM and GRM formulations. Here, we consider the transient vertical displacement at the receiver $\mathrm{A}\left(h^{01} / 2, h^{01} / 2\right)$ in the top subalyer of a laminate containing $N$ sublayers of the same thickness $h$, density $\rho$, and Poisson ratio $v$ impacted by a $F_{0} \delta(t) \delta(x)$ at the top surface. The Young modulus of the even sublayers is two times of that of the odd sublayers. Table I shows that the calculation time for GRM formulation increases much smaller than that for RRM formulation as the times of reflection or transmission $k$ increases.

The influence of the sublayer number $N$ on the calculation time of the vertical displacement at the receiver A for the eighth generalized ray group is shown in Table II. For the small $N$, the time consuming for GRM and RRM formulations are almost the same. Compared with GRM formulation, the calculation time for RRM formulation increases remarkably as the sublayer number $N$ increases, which yields the lower calculation efficiency. 


\begin{tabular}{|c|c|c|c|}
\hline & $\begin{array}{c}\mathrm{t}_{\mathrm{GRM}} \\
(\mathrm{s})\end{array}$ & $\begin{array}{c}\mathrm{t}_{\mathrm{RRM}} \\
(\mathrm{s})\end{array}$ & $\begin{array}{c}\mathrm{t}_{\mathrm{GRM}} / \mathrm{t}_{\mathrm{RRM}} \\
(\%)\end{array}$ \\
\hline $\mathbf{R}^{0}$ & 800 & 1147 & 69.7 \\
\hline $\mathbf{R}^{1}$ & 801 & 1206 & 66.4 \\
\hline $\mathbf{R}^{2}$ & 808 & 1271 & 63.6 \\
\hline $\mathbf{R}^{3}$ & 818 & 1319 & 62.0 \\
\hline $\mathbf{R}^{4}$ & 813 & 1361 & 59.7 \\
\hline $\mathbf{R}^{5}$ & 811 & 1414 & 57.4 \\
\hline $\mathbf{R}^{6}$ & 808 & 1436 & 56.3 \\
\hline $\mathbf{R}^{7}$ & 810 & 1494 & 54.2 \\
\hline
\end{tabular}

Table I. Calculation time of the transient vertical displacement at the receiver A $\left(h^{01}, h^{01} / 2\right)$ in the top sublayer of a ten-sublayered laminate

\begin{tabular}{|c|c|c|c|}
\hline & $\begin{array}{c}\mathrm{t}_{\text {GRM }} \\
(s)\end{array}$ & $\begin{array}{c}\mathrm{t}_{\text {RRM }} \\
(s)\end{array}$ & $\begin{array}{c}\mathrm{t}_{\text {GRM }} / \mathrm{t}_{\text {RRM }} \\
(\%)\end{array}$ \\
\hline$N=4$ & 486 & 539 & 90.2 \\
\hline$N=6$ & 605 & 830 & 72.9 \\
\hline$N=8$ & 724 & 1145 & 63.2 \\
\hline$N=10$ & 810 & 1494 & 54.2 \\
\hline$N=12$ & 959 & 2000 & 48.0 \\
\hline$N=14$ & 1077 & 2565 & 42.0 \\
\hline$N=16$ & 1200 & 3203 & 37.5 \\
\hline$N=18$ & 1318 & 4006 & 32.9 \\
\hline$N=20$ & 1439 & 4886 & 29.5 \\
\hline
\end{tabular}

Table II. Calculation time of the transient vertical displacement for $\mathbf{R}^{7}$ at the receiver $\mathrm{A}\left(h^{01}\right.$, $h^{01} / 2$ ) in the top sublayer of a $N$-sublayered laminate 


\section{Conclusions}

In conclusion, we present the formulations of the reverberation-ray matrix, reverberationtransfer matrix, and generalized reverberation matrix clearly. Their comparison shows that the application of the RRM formulation to the receiving sublayer and the SM and TM formulations to the other sublayers in the GRM and RTM methods yields a generalized reverberation matrix of the constant dimension, which is independent of the sublayer number. But RTM has the numerical instability for the large product of frequency and thickness, which means that it is only suitable for the low-frequency response in the multilayered solids. The numerical examples show that the calculating time for transient wave propagation in GRM formulation increases much smaller than that for RRM formulation as the times of reflection or transmission $k$ and the sublayer number $N$ increase, which promises the higher calculation efficiency of the generalized rays in the complex multilayered configuration compared with RRM formulation.

\section{Acknowledgements}

A part of this study was supported by the National Natural Science Foundation of China (No. 10602053 and No. 50808170), research grants from Institute of Crustal Dynamics (No. ZDJ2007-2) and for oversea-returned scholar, Personnel Ministry of China.

\section{References}

Achenbach, J. D. (1973). "Wave propagation in elastic solids," North-Holland, Amsterdam.

Haskell, N. A. (1953). the dispersion of surface waves on multilayered media. Bulletin of the Seismological Society of America 43, 17-34.

Lowe, M. J. S. (1995). Matrix techniques for modeling ultrasonic waves in multilayered media. IEEE Transactions on Ultrasonics, Ferroelectrics, and Frequency Control 42, 525542.

Pao, Y. H., Su, X. Y., and Tian, J. Y. (2000). Reverberation matrix method for propagation of sound in a multilayered liquid. Journal of Sound and Vibration 230, 743-760.

Rokhlin, S. I., and Wang, L. (2002). Stable recursive algorithm for elastic wave propagation in layered anisotropic media: Stiffness matrix method. Journal of the Acoustical Society of America 112, 822-834.

$\mathrm{Su}$, X. Y., Tian, J. Y., and Pao, Y. H. (2002). Application of the reverberation-ray matrix to the propagation of elastic waves in a layered solid. International Journal of Solids and Structures 39, 5447-5463.

Thomson, T. (1950). Transmission of elastic waves through a stratified solid medium. Journal of Applied Physics 21, 89-93.

Tian, J., and Xie, Z. (2009). A hybrid method for transient wave propagation in a multilayered solid. Journal of Sound and Vibration 325, 161-173.

Tian, J. Y., Yang, W. X., and Su, X. Y. (2006). Transient elastic waves in a transversely isotropic laminate impacted by axisymmetric load. Journal of Sound and Vibration 289, 94-108. 
Wang, L., and Rokhlin, S. I. (2001). Stable reformulation of transfer matrix method for wave propagation in layered anisotropic media. Ultrasonics 39, 413-424. 


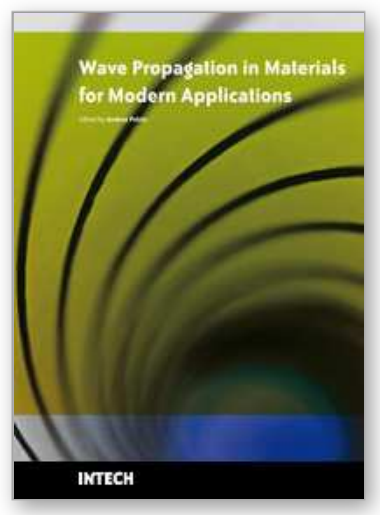

\section{Wave Propagation in Materials for Modern Applications}

Edited by Andrey Petrin

ISBN 978-953-7619-65-7

Hard cover, 526 pages

Publisher InTech

Published online 01, January, 2010

Published in print edition January, 2010

In the recent decades, there has been a growing interest in micro- and nanotechnology. The advances in nanotechnology give rise to new applications and new types of materials with unique electromagnetic and mechanical properties. This book is devoted to the modern methods in electrodynamics and acoustics, which have been developed to describe wave propagation in these modern materials and nanodevices. The book consists of original works of leading scientists in the field of wave propagation who produced new theoretical and experimental methods in the research field and obtained new and important results. The first part of the book consists of chapters with general mathematical methods and approaches to the problem of wave propagation. A special attention is attracted to the advanced numerical methods fruitfully applied in the field of wave propagation. The second part of the book is devoted to the problems of wave propagation in newly developed metamaterials, micro- and nanostructures and porous media. In this part the interested reader will find important and fundamental results on electromagnetic wave propagation in media with negative refraction index and electromagnetic imaging in devices based on the materials. The third part of the book is devoted to the problems of wave propagation in elastic and piezoelectric media. In the fourth part, the works on the problems of wave propagation in plasma are collected. The fifth, sixth and seventh parts are devoted to the problems of wave propagation in media with chemical reactions, in nonlinear and disperse media, respectively. And finally, in the eighth part of the book some experimental methods in wave propagations are considered. It is necessary to emphasize that this book is not a textbook. It is important that the results combined in it are taken "from the desks of researchers". Therefore, I am sure that in this book the interested and actively working readers (scientists, engineers and students) will find many interesting results and new ideas.

\section{How to reference}

In order to correctly reference this scholarly work, feel free to copy and paste the following:

Jiayong Tian (2010). Comparison Between Reverberation-Ray Matrix, Reverberation-Transfer Matrix, and Generalized Reverberation Matrix, Wave Propagation in Materials for Modern Applications, Andrey Petrin (Ed.), ISBN: 978-953-7619-65-7, InTech, Available from: http://www.intechopen.com/books/wave-propagationin-materials-for-modern-applications/comparison-between-reverberation-ray-matrix-reverberation-transfermatrix-and-generalized-reverberat

\section{INTECH}

open science | open minds

InTech Europe

InTech China 
University Campus STeP Ri Slavka Krautzeka 83/A 51000 Rijeka, Croatia Phone: +385 (51) 770447 Fax: +385 (51) 686166 www.intechopen.com
Unit 405, Office Block, Hotel Equatorial Shanghai No.65, Yan An Road (West), Shanghai, 200040, China 中国上海市延安西路65号上海国际贵都大饭店办公楼405单元 Phone: +86-21-62489820

Fax: +86-21-62489821 
(C) 2010 The Author(s). Licensee IntechOpen. This chapter is distributed under the terms of the Creative Commons Attribution-NonCommercialShareAlike-3.0 License, which permits use, distribution and reproduction for non-commercial purposes, provided the original is properly cited and derivative works building on this content are distributed under the same license. 